\title{
Complications of nasal and pharyngeal swabs: a relevant challenge of the COVID-19 pandemic?
}

\author{
To the Editor:
}

The coronavirus disease 2019 (COVID-19) pandemic comprises approximately 50 million confirmed cases and over 1.2 million deaths as of 10 November, 2020 [1], affecting healthcare systems worldwide in an unprecedented way. In the absence of effective treatments or preventive measures, all attempts to control the pandemic are based on reliable diagnostic procedures, particularly RT-PCR of upper respiratory specimens, which is considered the diagnostic gold standard [2]. A previously unimaginable number of these diagnostic procedures has been performed since the beginning of the pandemic and there is a clear trend towards further expanding the number of tests [3]. Although specimens are frequently obtained by semi-skilled temporary staff, the collection is generally considered safe. However, possible adverse events of the procedure have largely escaped systematic recording and reporting to date. A Pubmed search, performed on 10 October, 2020, using every possible combination of the search terms "complications", "adverse events", "adverse effects" and "nasal swab", "oral swab", "nasopharyngeal swab", "oropharyngeal swab", revealed only three publications relevant for the question of adverse events caused by pharyngeal swab procedures. The first one represents a case report describing the break of a nasal swab by triggering the swab's breakpoint mechanism during the examination of an uncooperative patient [4]. The second publication compared commercially available swabs with three-dimensional printed nasopharyngeal swabs, reporting different mild complications in several individuals, and one individual with severe epistaxis needing medical help [5]. Lastly, one case of cerebrospinal fluid leak requiring endoscopic surgical repair was reported after a nasal COVID-19 test [6]. Of note, even the second study investigated adverse events in only 176 individuals [5].

Here, we evaluated complications caused by deep nasal and oropharyngeal swabs requiring immediate medical attention in a large, representative cohort from Northern Germany and estimated the number of tests for severe acute respiratory syndrome coronavirus 2 (SARS-CoV-2) involving swab procedures during the pandemic worldwide.

Within our population-based SARS-CoV-2 monitoring study (ELISA cohort), 11476 deep nasal and oropharyngeal swabs were taken in 3083 individuals from May to August 2020. Swab collection followed a clinically approved protocol as performed at the University Hospital Schleswig-Holstein, Campus Lübeck, Germany, and consisted of combined deep nasal (mid-turbinate) and oropharyngeal swabs in each participant. The deep nasal swab was performed by inserting the swab $2-3 \mathrm{~cm}$ into one nostril (until resistance was felt at the turbinates), while gently rotating. The same swab was then inserted through the mouth and rubbed between the tonsillar pillars over the posterior oropharynx avoiding the tongue, teeth, and gums. CE-marked PROBACT Transport Swabs (Technical Service Consultants Ltd., UK) and CITOSWAB Transport Swabs (Citotest Scientific, China) were used throughout the study. Specially trained medical students performed the swabs under the on-site supervision of a medical doctor.

Adverse events were documented in a standardised manner and classified as situations requiring immediate medical diagnosis or treatment, whereas severe adverse events were considered study-related complications causing permanent damage.

@ERSpublications

Although swab procedures during SARS-CoV-2 testing are generally safe (3 adverse events in 11476 swab procedures; $0.026 \%$ ), increased awareness of complications is necessary, considering approximately 5.1 million tests conducted worldwide daily. https://bit.ly/2J5sd 96

Cite this article as: Föh $\mathrm{B}$, Borsche $\mathrm{M}$, Balck $\mathrm{A}$, et al. Complications of nasal and pharyngeal swabs: a relevant challenge of the COVID-19 pandemic?. Eur Respir J 2021; 57: 2004004 [https://doi.org/10.1183/ 13993003.04004-2020]. 
TABLE 1 Number of swab procedures for detection of severe acute respiratory syndrome coronavirus 2 until 13 November, 2020

\begin{tabular}{lcccc} 
Continent & $\begin{array}{c}\text { Countries } \\
\text { included }\end{array}$ & $\begin{array}{c}\text { Population included } \\
\text { (million) }\end{array}$ & $\begin{array}{c}\text { Tests total (million) } \\
\text { Tests } \\
\text { daily }\end{array}$ \\
\hline Africa & 18 & 731 & 15.0 & 74584 \\
Asia & 25 & 2742 & 219.8 & 1806250 \\
Europe & 32 & 642 & 213.2 & 1989026 \\
North America & 9 & 546 & 175.9 & 1085027 \\
Oceania & 3 & 31 & 10.4 & 40.9 \\
South America & 6 & 110 & 10582 & 75013 \\
Total & 93 & 4807 & 645.2 & 5076752 \\
\hline
\end{tabular}

Based on World Health Organization data (source: https:/github.com/owid/covid-19-data/tree/master/ public/data/ as accessed on 16 November, 2020). \#: cumulative number of tests since March 2020, latest value period 2-13 November, 2020; १: daily number of tests, latest value period 2-13 November, 2020, smoothed (7 days).

We observed a total of three adverse events $(0.026 \%$, 95\% CI $0.007-0.077 \%)$. In two individuals, a 53 -year-old and a 55-year-old male, the swab's tip broke off. In both cases, the swab tip was not visible by inspection. While the first person had a foreign body sensation, the second person did not report any similar complaints. Both individuals were immediately transferred to an otorhinolaryngology clinic, where the swab tip was retrieved without complications by nasal endoscopy in the first individual. The swab tip was no longer detectable in the second person despite a thorough examination by an otorhinolaryngologist, suggesting that the tip had been swallowed without further complications. Third, a 29-year-old female developed a spontaneous anterior dislocation of the left temporomandibular joint when opening her mouth for the oropharyngeal swab. Exhibiting relevant pain, she was admitted to a hospital by ambulance for external jaw repositioning. Notably, no individual developed epistaxis that would have required medical care, nor were there any severe adverse events.

According to World Health Organization data, at least 645 million tests involving swab procedures have been performed since the beginning of the pandemic until 13 November, 2020. In the first half of November 2020, the total number of daily tests amounted to 5.1 million (table 1). Of note, the observed occurrence of adverse events in $0.026 \%$ of swab procedures is possibly still an underestimate, as we performed a combination of deep nasal and oropharyngeal swabs that yield comparable virus detection rates [7], but are less invasive than nasopharyngeal swabs.

Our results from a well-monitored, large cohort demonstrate that the combination of deep nasal and oropharyngeal swabs is generally safe. Adverse events are very rare, severe adverse events are unlikely, but they cannot be entirely ruled out. However, given the exceptionally high number of SARS-CoV-2 tests performed worldwide that involve an increasing number of healthy, asymptomatic individuals with very low a priori probabilities for acute infections $[8,9]$, adverse events of the diagnostic procedure require appropriate attention, as is common practice in SARS-CoV-2 drug and vaccine development.

In conclusion, we would like to encourage: 1) increased awareness for adverse events during the standard medical procedure for SARS-CoV-2 testing; 2) the provision of appropriate diagnostic and therapeutic measures in case of adverse events; and 3) further research addressing adverse events, including the need to implement appropriate informed consent in clinical as well as in research settings.

Bandik Föh $\oplus^{1,6}$, Max Borsche ${ }^{2,6}$, Alexander Balck ${ }^{2}$, Stefan Taube ${ }^{3}$, Jan Rupp ${ }^{4}$, Christine Klein ${ }^{2}$ and Alexander Katalinic $\mathbb{1}^{5}$

${ }^{1}$ Institute of Nutritional Medicine, University of Lübeck, Lübeck, Germany. ${ }^{2}$ Institute of Neurogenetics, University of Lübeck, Lübeck, Germany. ${ }^{3}$ Institute of Virology and Cell Biology, University of Lübeck, Lübeck, Germany. ${ }^{4}$ Dept of Infectious Diseases and Microbiology, University of Lübeck, Lübeck, Germany. ${ }^{5}$ Institute of Social Medicine and Epidemiology, University of Lübeck, Lübeck, Germany. ${ }^{6}$ Shared first authorship, equal contributions.

Correspondence: Christine Klein, Institute of Neurogenetics, University of Lübeck, BMF, Building 67, Ratzeburger Allee 160, 23538 Lübeck, Germany. E-mail: christine.klein@neuro.uni-luebeck.de

Received: 29 Oct 2020 | Accepted: 18 Nov 2020

Author contributions: All authors contributed to the study concept and design. B. Föh, M. Borsche, A. Balck and S. Taube contributed to the acquisition of data. B. Föh, M. Borsche, C. Klein and A. Katalinic contributed to the analysis and interpretation of data. B. Föh and M. Borsche contributed to drafting the manuscript. S. Taube, C. Klein 
and A. Katalinic contributed to critically revising the manuscript for important intellectual content. B. Föh and M. Borsche contributed equally. All authors read and approved the final manuscript.

Conflict of interest: B. Föh has nothing to disclose. M. Borsche has nothing to disclose. A. Balck has nothing to disclose. $\mathrm{S}$. Taube has nothing to disclose. J. Rupp has nothing to disclose. C. Klein reports personal fees from Centogene GmbH, outside the submitted work. A. Katalinic reports grants from State of Schleswig-Holstein, Germany and the Federal Ministry of Education and Research, Germany, during the conduct of the study.

Support statement: The main study was financially supported by the State of Schleswig-Holstein, the Federal Ministry of Education and Research (BMBF), and additional crowdfunding by the University of Lübeck. B. Föh's position was supported by the German Research Foundation (IRTG 1911). Funding information for this article has been deposited with the Crossref Funder Registry.

\section{References}

1 World Health Organization. Coronavirus Disease (COVID-19) Weekly Epidemiological Update November 10, 2020. www.who.int/docs/default-source/coronaviruse/situation-reports/20201110-weekly-epi-update-13.pdf?sfvrsn=244354 77_15\&download=true Date last updated: 10 Nov 2020. Date last accessed: 11 Nov 2020.

2 Wiersinga WJ, Rhodes A, Cheng AC, et al. Pathophysiology, transmission, diagnosis, and treatment of coronavirus disease 2019 (COVID-19): a review. JAMA 2020; 324: 782-793.

3 Our World in Data. Coronavirus Pandemic (COVID-19) - Statistics and Research. https://ourworldindata.org/ coronavirus?utm_campaign=Optimizando\&utm_medium=email\&utm_source=Revue newsletter Date last updated: 20 Oct 2020. Date last accessed: 20 Oct 2020.

4 Mughal Z, Luff E, Okonkwo O, et al. Test, test, test - a complication of testing for coronavirus disease 2019 with nasal swabs. J Laryngol Otol 2020; 134: 646-649.

5 Callahan CJ, Lee R, Zulauf KE, et al. Open development and clinical validation of multiple 3d-printed nasopharyngeal collection swabs: rapid resolution of a critical covid-19 testing bottleneck. J Clin Microbiol 2020; 58: e00876-20.

6 Sullivan CB, Schwalje AT, Jensen M, et al. Cerebrospinal fluid leak after nasal swab testing for coronavirus disease 2019. JAMA Otolaryngol Neck Surg 2020; 146: 1179-1181.

7 LeBlanc JJ, Heinstein C, MacDonald J, et al. A combined oropharyngeal/nares swab is a suitable alternative to nasopharyngeal swabs for the detection of SARS-CoV-2. J Clin Virol 2020; 128: 104442.

8 Gandhi M, Yokoe DS, Havlir DV. Asymptomatic transmission, the Achilles' heel of current strategies to control Covid-19. N Engl J Med 2020; 382: 2158-2160.

9 Treibel TA, Manisty C, Burton M, et al. COVID-19: PCR screening of asymptomatic health-care workers at London hospital. Lancet 2020; 395: 1608-1610. 